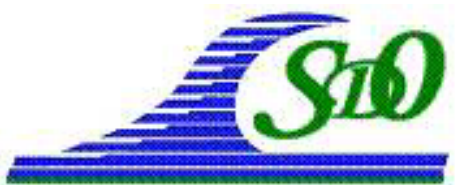

XI ${ }^{\text {èmes }}$ Journées Nationales Génie Côtier - Génie Civil

Les Sables d'Olonne, 22-25 juin 2010

DOI:10.5150/jngcgc.2010.013-P C Editions Paralia CFL

disponible en ligne - http://www.paralia.fr - available online

\title{
Étude d'agitation dans le bassin de Port 2000
}

\section{Céline PERHERIN ${ }^{1}$}

\author{
1. Centre d'Études Techniques Maritimes et Fluviales, DELCE, DHSM, \\ BP 5, 29280 Plouzané, France. \\ Celine.Perherin@developpement-durable.gouv.fr
}

\section{Résumé :}

Une connexion est envisagée entre le port à conteneurs du Havre, Port 2000, et le réseau des voies navigables intérieures au moyen d'une écluse implantée en fond de darse. Les conditions d'agitation du bassin doivent être bien connues afin de savoir si les unités fluviales y sont adaptées et de connaître le pourcentage moyen d'indisponibilité des postes à quai.

Le CETMEF a été chargé de réaliser cette étude pour laquelle une méthode d'évaluation de l'agitation a été mise au point. L'agitation sur le bassin Port 2000 peut être liée à la fois aux houles en provenance du large et aux clapots levés sur le bassin d'une longueur de plus de $5 \mathrm{~km}$. Les impacts des houles du large et des clapots ont été évalués indépendamment pour chaque poste à quai. Une méthode d'évaluation de l'agitation liée à la concomitance des deux phénomènes a également été développée dans l'objectif d'évaluer par excès la probabilité d'indisponibilité des postes à quai pour les navettes fluviales.

\section{Mots-clés :}

Agitation - Houle - Clapot - Modélisation - Combinaison - Probabilités

\begin{abstract}
:
A connection between the maritime harbour of Le Havre, Port 2000, and the network of inland waterways is planned. Wave agitation has to be taken in account in order to know if inland waterways ships are adapted to those conditions and to estimate the average percentage of unavailability of every dock.

CETMEF produced this study for the Grand Port Maritime du Havre. A method for the estimation of wave agitation has been developed. Wave agitation at Port 2000 is linked to swell and wind-waves generated on the $5 \mathrm{~km}$-long basin. Impacts of swell and windwaves have been separately estimated. A method to estimate wave agitation linked to the simultaneity of swell and wind-waves has been developed in order to assess by excess the average percentage of ship-unavailability of every dock.
\end{abstract}

\section{Keywords:}

Wave agitation - Swell - Wind-waves - Modelling - Simultaneity 


\section{Introduction}

Une connexion est envisagée entre le port à conteneurs du Havre, Port 2000, et le réseau des voies navigables intérieures au moyen d'une écluse implantée en fond de darse (voir figure 1). Les unités fluviales pourront ainsi atteindre les postes à quai, de 350 mètres chacun, sans être exposées aux conditions de navigation extérieures au bassin.

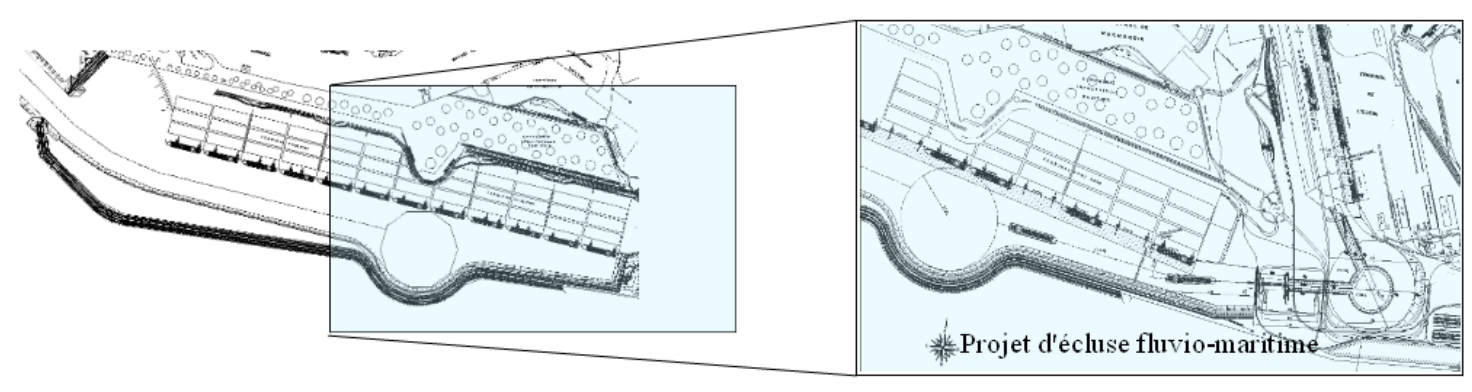

Figure 1. Bassin de Port 2000 et projet d'écluse fluvio-maritime (Source : Grand Port Maritime du Havre).

L'étude réalisée (PERHERIN, 2008) avait pour objectif d'évaluer l'agitation totale, combinaison de l'impact des houles et du clapot levé par le vent, sur chacun des 12 postes à quai du bassin de Port 2000 et d'estimer l'indisponibilité de chacun des postes à quai aux unités fluviales pour différents seuils. Une méthodologie d'évaluation de l'agitation totale a été élaborée et mise en œuvre sur l'ensemble du bassin. Cette application s'est basée sur les résultats de deux rapports d'études précédents du CETMEF pour lesquels une méthode d'évaluation de l'impact des houles d'une part (LE GUYADER, 2007) et une méthode d'évaluation de l'impact du clapot d'autre part (LEBRETON et al., 2006) avaient été élaborées.

\section{Méthodologie globale de l'étude}

L'objectif de l'étude était de connaître l'agitation globale du bassin et d'en déduire l'indisponibilité annuelle de chacun des postes à quai, c'est-à-dire la probabilité que l'agitation soit supérieure au seuil de navigabilité des unités fluviales. D'après les prescriptions existantes en matière de navigation intérieure, le seuil de navigabilité correspondrait à des hauteurs significatives allant de $0,5 \mathrm{~m}$ à $1 \mathrm{~m}$ (LEBRETON et al., 2006). Trois seuils d'agitation $S$, qui sont assimilés à la hauteur significative $H_{s}$, ont été testés : $\mathrm{S}_{1}=1,00 \mathrm{~m}, \mathrm{~S}_{2}=0,75 \mathrm{~m}$ et $\mathrm{S}_{3}=0,50 \mathrm{~m}$. Les probabilités que l'agitation soit supérieure à chaque seuil et comprise dans chacune des classes $[0,50 ; 0,75 \mathrm{~m}]$, $[0,75 ; 1,00 \mathrm{~m}]$ et $[1,00 \mathrm{~m} ;+\infty[$ ont été déterminées.

L'agitation pouvant intervenir dans le bassin Port 2000 est principalement liée à deux phénomènes : l'impact de la houle du large et du clapot levé par le vent sur le bassin. L'agitation totale $\mathrm{Ht}$ dans le bassin peut dépasser un seuil $\mathrm{S}$ lorsque : 
- la houle du large pénètre dans le bassin et génère une agitation de hauteur Hh supérieure à $\mathrm{S}$ (événement $\mathrm{H}$ ),

- le vent lève un clapot de hauteur Hc supérieure à S (événement C),

- la concomitance des deux phénomènes génère une agitation résultante $\mathrm{Hr}$, avec $\mathrm{Hr}=\sqrt{ }\left(\mathrm{Hc}^{2}+\mathrm{Hh}^{2}\right)$, supérieure à $\mathrm{S}$ alors que $\mathrm{Hh}$ et $\mathrm{Hc}$ sont inférieures à $\mathrm{S}$, c'est à dire que : $\mathrm{Hr}>\mathrm{S}$ et $\mathrm{Hc}<\mathrm{S}$ et $\mathrm{Hh}<\mathrm{S}$ (événement $\mathrm{R}$ ).

La probabilité que l'agitation totale $\mathrm{Ht}$ soit supérieure à un seuil $\mathrm{S}$ est donc :

$\mathrm{P}(\mathrm{Ht}>\mathrm{S})=\mathrm{P}(\mathrm{H} \cup \mathrm{C} \cup \mathrm{R})$

$\mathrm{P}(\mathrm{Ht}>\mathrm{S})=\mathrm{P}(\mathrm{H} \cup \mathrm{C})+\mathrm{P}(\mathrm{R})$

$\mathrm{P}(\mathrm{Ht}>\mathrm{S})=\mathrm{P}(\mathrm{H})+\mathrm{P}(\mathrm{C})-\mathrm{P}(\mathrm{H} \cap \mathrm{C})+\mathrm{P}(\mathrm{R})$

$\mathrm{P}(\mathrm{H}>\mathrm{S})<\mathrm{P}(\mathrm{H})+\mathrm{P}(\mathrm{C})+\mathrm{P}(\mathrm{R})$

Les phénomènes de houle et de clapot ne sont en effet pas indépendants. Une analyse des séries temporelles communes du vent et de la houle aurait permis d'évaluer la probabilité $\mathrm{P}(\mathrm{H} \cap \mathrm{C})$. Dans la présente étude, une évaluation par excès de la probabilité que l'agitation soit supérieure à un seuil $\mathrm{S}$ a été retenue.

Pour calculer les probabilités d'une agitation supérieure au seuil S, doivent ainsi être préalablement déterminées :

- les probabilités que le clapot levé par le vent et l'impact de la houle du large appartiennent à chacune des classes $[0,00 ; 0,25 \mathrm{~m}],[0,25 ; 0,50 \mathrm{~m}],[0,50 ; 0,75 \mathrm{~m}]$, $[0,75 ; 1,00 \mathrm{~m}]$ et $\left[1,00 \mathrm{~m} ;+\infty\left[\right.\right.$ (probabilités des événements $H_{\mathrm{i}}$ et $\left.\mathrm{C}_{\mathrm{i}} 1<\mathrm{i}<5\right)($ voir tableau 1),

- les probabilités $\mathrm{P}(\mathrm{R} 1), \mathrm{P}(\mathrm{R} 2)$ et $\mathrm{P}(\mathrm{R} 3)$ que la hauteur résultant de la concomitance de deux phénomènes (chacun étant inférieur au seuil) soit supérieure aux 3 seuils,

- les probabilités de concomitance de chaque classe de houle et de clapot $(\mathrm{P}(\mathrm{C} 2 \cap \mathrm{H} 2)$, $\mathrm{P}(\mathrm{C} 2 \cap \mathrm{H} 3) \ldots)$ (voir tableau 1).

Tableau 1. Tableau de définition des événements.

\begin{tabular}{llllll}
\hline & $H c$ & $C 1:$ & $C 2:$ & $C 3:$ & $C 4:$ \\
$H h$ & $1,00<H c$ & $0,75<H c<1,00$ & $0,50<H c<0,75$ & $0,25<H c<0,50$ & $\begin{array}{l}C 5 \\
0<H c<0,25\end{array}$ \\
\hline$H 1: 1,00<H h$ & $C 1 \cap H 1$ & $C 2 \cap H 1$ & $C 3 \cap H 1$ & $C 4 \cap H 1$ & $C 5 \cap H 1$ \\
$H 2: 0,75<H h<1,00$ & $C 1 \cap H 2$ & $C 2 \cap H 2$ & $C 3 \cap H 2$ & $C 4 \cap H 2$ & $C 5 \cap H 2$ \\
$H 3: 0,50<H h<0,75$ & $C 1 \cap H 3$ & $C 2 \cap H 3$ & $C 3 \cap H 3$ & $C 4 \cap H 3$ & $C 5 \cap H 3$ \\
$H 4: 0,25<H h<0,50$ & $C 1 \cap H 4$ & $C 2 \cap H 4$ & $C 3 \cap H 4$ & $C 4 \cap H 4$ & $C 5 \cap H 4$ \\
$H 5: 0<H h<0,25$ & $C 1 \cap H 5$ & $C 2 \cap H 5$ & $C 3 \cap H 5$ & $C 4 \cap H 5$ & $C 5 \cap H 5$ \\
\hline
\end{tabular}

\section{Clapot levé par le vent}

La longueur du bassin de Port 2000, de la passe d'entrée à l'est à l'entrée de la future écluse à l'ouest, est supérieure à $5 \mathrm{~km}$ (5300 mètres) et sa largeur est comprise entre 250 et $700 \mathrm{~m}$. Cette longueur est suffisante pour lever des clapots significatifs lorsque le 
vent souffle dans l'axe du bassin. La méthode retenue pour le calcul du clapot levé par le vent sur l'ensemble de la darse est la méthode de DONELAN (DONELAN, 1980 ; BISHOP \& DONELAN, 1987 ; BISHOP et al., 1992 ; DONELAN et al., 1992). Elle permet l'évaluation du clapot levé par un vent oblique. Cette méthode est fondée sur le fait que la longueur de fetch peut être mesurée suivant la direction des vagues, considérée comme l'axe longitudinal du plan d'eau, plutôt que suivant la direction du vent. La formule utilisée pour la détermination de la hauteur de houle $\mathrm{H}_{\mathrm{S}}$ est :

$$
\begin{aligned}
& \frac{g H_{s i}}{\left(U_{10} \cos \left(\theta-\varphi_{w}\right)\right)^{2}}=0.00366\left(\frac{g F_{\theta}}{\left(U_{10} \cos \left(\theta-\varphi_{w}\right)\right)^{2}}\right)^{0.38 \quad \text { avec : } \mathrm{F}(\mathrm{m}) \text { le fetch }} \begin{array}{c}
\mathrm{U}_{10}(\mathrm{~m} / \mathrm{s}) \text { la vitesse du vent à } 10 \mathrm{~m} \\
\theta\left({ }^{\circ}\right) \text { la direction des vagues }
\end{array} \\
& \theta\left({ }^{\circ}\right) \text { la direction des vagues } \\
& \phi_{w}\left(^{\circ}\right) \text { la direction du vent }
\end{aligned}
$$

Les secteurs de vent retenus pouvant provoquer du clapot sont $\left[230^{\circ} ; 330^{\circ}\right]$ et $\left[50^{\circ}\right.$; $150^{\circ}$ ] (PERHERIN, 2008). Pour chaque poste à quai et direction (par secteur de $20^{\circ}$ ), l'intensité du vent provoquant un clapot supérieur à chaque seuil a été calculée. A partir des statistiques directionnelles de vent et de la connaissance des queues de distribution d'intensité du vent, les fréquences associées à la hauteur de clapot ont pu être évaluées. Les probabilités d'obtenir une agitation liée au clapot supérieure à un seuil, puis les probabilités de chaque classe de clapot, ont été calculées par poste à quai par somme des différentes probabilités par secteur directionnel de vent (voir tableau 2).

\begin{tabular}{|c|c|c|c|c|c|c|c|}
\hline $\begin{array}{l}\text { Provenance du vent } \\
\text { Hauteur }\end{array}$ & $240^{\circ}$ & $260^{\circ}$ & $280^{\circ}$ & $300^{\circ}$ & $320^{\circ}$ & $\begin{array}{l}{\left[50^{\circ} ;\right.} \\
\left.150^{\circ}\right]\end{array}$ & Total \\
\hline $1,00 \mathrm{~m}$ & 0,001 & 0,009 & 0,018 & 0,006 & 0 & 0 & 0,034 \\
\hline$[1,00 \mathrm{~m}, \infty \Gamma$ & 0,001 & 0,009 & 0,018 & 0,006 & 0 & 0 & 0,034 \\
\hline $0,75 \mathrm{~m}$ & 0,013 & 0,075 & 0,101 & 0,031 & 0,003 & 0 & 0,223 \\
\hline$[0,75 \mathrm{~m}, 1,00 \mathrm{~m}]$ & 0,012 & 0,066 & 0,083 & 0,025 & 0,003 & 0 & 0,189 \\
\hline $0,50 \mathrm{~m}$ & 0,192 & 0,647 & 0,607 & 0,226 & 0,071 & 0,008 & 1,751 \\
\hline$[0,50 \mathrm{~m}, 0,75 \mathrm{~m}]$ & 0,179 & 0,572 & 0,506 & 0,195 & 0,068 & 0,008 & 1,528 \\
\hline $0,25 \mathrm{~m}$ & 2,530 & 2,901 & 2,675 & 1,232 & 0,639 & 1,699 & 11,676 \\
\hline$[0,25 \mathrm{~m}, 0,50 \mathrm{~m}]$ & 2,338 & 2,254 & 2,068 & 1,006 & 0,568 & 1,691 & 9,925 \\
\hline$[0 \mathrm{~m}, 0,25 \mathrm{~m}]$ & & & & & & & 88,324 \\
\hline
\end{tabular}

Tableau 2. Probabilités (\%) qu'un clapot soit supérieur à un seuil donné et compris dans une classe d'agitation sur le poste $n^{\circ} 6$.

\section{Impact des houles du large}

La contribution de l'impact des houles à l'agitation totale du bassin de Port 2000 est importante (LE GUYADER et al., 2007). Les houles en provenance du secteur directionnel $\left[270^{\circ} ; 330^{\circ}\right]$ sont les principales responsables de l'agitation à l'intérieur du bassin. L'évaluation de l'impact des houles a été réalisée par le biais de propagations avec le modèle REFONDE sur le cluster du CETMEF. Les statistiques directionnelles 


\section{XI $I^{\text {èes }}$ Journées Nationales Génie Côtier - Génie Civil}

Les Sables d'Olonne, 22-25 juin 2010

de houles proviennent de l'analyse des mesures de houles du Valeport 730 mouillé de septembre 2002 à décembre 2004 à $1 \mathrm{~km}$ à l'ouest du caisson-musoir sud de la passe d'entrée de Port 2000. Des propagations à niveau constant (pleine mer de vive eau) ont été réalisées pour trois secteurs directionnels de $20^{\circ}\left(280^{\circ}, 300^{\circ}\right.$ et $\left.320^{\circ}\right)$ et pour une houle incidente $\mathrm{H}_{\mathrm{s}}$ comprise entre $0,5 \mathrm{~m}$ et $3 \mathrm{~m}$ (avec un pas de $25 \mathrm{~cm}$ ).

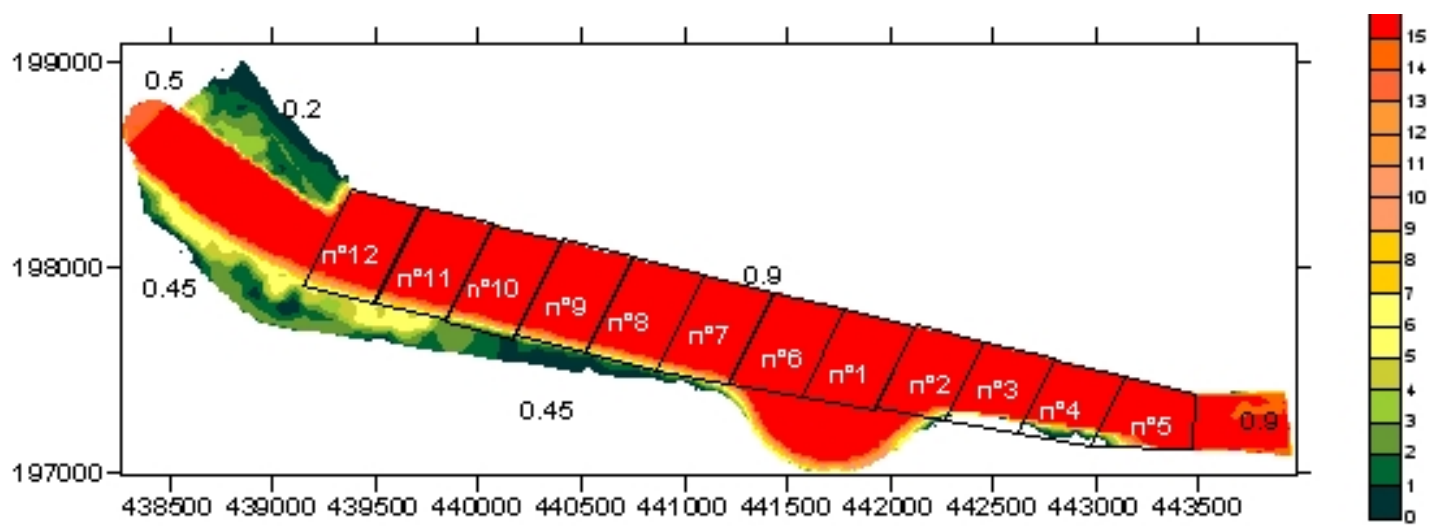

Figure 2. Bathymétrie, postes à quai et coefficients de réflexion des ouvrages.

La probabilité que l'agitation liée aux houles soit supérieure à chaque seuil a été évaluée pour chaque poste à quai. Pour un poste à quai, l'agitation retenue est le quantile à $95 \%$ de l'ensemble de la surface du poste à quai considéré (voir figures 2 et 3), le posttraitement étant réalisé grâce au logiciel SURFER. A partir des statistiques directionnelles de houles, les fréquences associées à la hauteur de l'agitation ont pu être évaluées. Les probabilités d'obtenir une agitation liée aux houles supérieure à un seuil, puis les probabilités de chaque classe d'agitation, ont été calculées par poste à quai par somme des différentes probabilités par secteur directionnel (voir tableau 3).

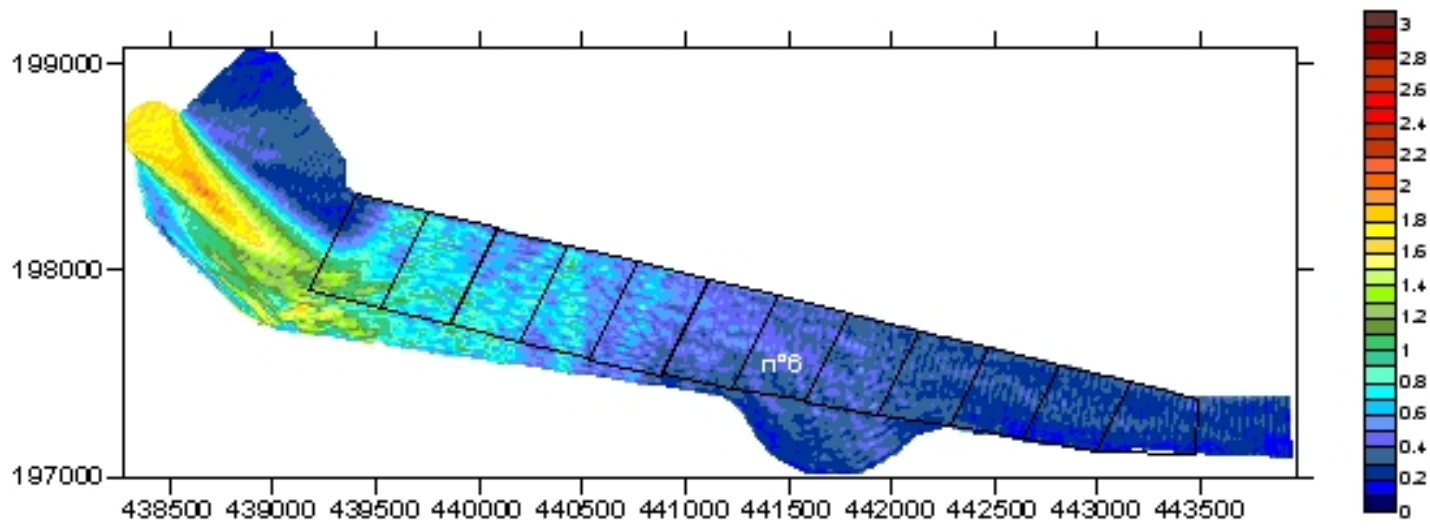

Figure 3. Impact d'une houle de $H s=2,5 \mathrm{~m}$ de provenance $320^{\circ}$, poste à quai 6 . 
Thème 1 - Hydrodynamique côtière

Tableau 3. Probabilités (\%) que l'impact des houles soit supérieur à un seuil donné et compris dans une classe d'agitation sur le poste $n^{\circ} 6$.

\begin{tabular}{|c|c|c|c|c|}
\hline $\begin{array}{l}\text { Provenance des houles } \\
\text { Hauteur }\end{array}$ & $280^{\circ}$ & $300^{\circ}$ & $320^{\circ}$ & Total \\
\hline $\begin{array}{l}1,00 \mathrm{~m} \\
{[1,00 \mathrm{~m}, \infty]}\end{array}$ & $\begin{array}{l}0 \\
0\end{array}$ & $\begin{array}{l}0,085 \\
0,085\end{array}$ & $\begin{array}{l}0 \\
0\end{array}$ & $\begin{array}{l}0,085 \\
0,085\end{array}$ \\
\hline $\begin{array}{l}0,75 \mathrm{~m} \\
{[0,75 \mathrm{~m}, 1,00 \mathrm{~m}]}\end{array}$ & $\begin{array}{l}0 \\
0\end{array}$ & $\begin{array}{l}0,245 \\
0,160\end{array}$ & $\begin{array}{l}0 \\
0\end{array}$ & $\begin{array}{l}0,245 \\
0,160\end{array}$ \\
\hline $\begin{array}{l}0,50 \mathrm{~m} \\
{[0,50 \mathrm{~m}, 0,75 \mathrm{~m}]}\end{array}$ & $\begin{array}{l}0,085 \\
0,085\end{array}$ & $\begin{array}{l}1,412 \\
1,167\end{array}$ & $\begin{array}{l}0,160 \\
0,160\end{array}$ & $\begin{array}{l}1,659 \\
1,412\end{array}$ \\
\hline $\begin{array}{l}0,25 \mathrm{~m} \\
{[0,25 \mathrm{~m}, 0,50 \mathrm{~m}]}\end{array}$ & $\begin{array}{l}1,176 \\
1,091\end{array}$ & $\begin{array}{l}9,538 \\
8,126\end{array}$ & $\begin{array}{l}0,913 \\
0,753\end{array}$ & $\begin{array}{l}11,627 \\
9,970\end{array}$ \\
\hline$[0 \mathrm{~m}, 0,25 \mathrm{~m}]$ & & & & 88,373 \\
\hline
\end{tabular}

\section{Concomitance houle/clapot}

Lorsque Hh et Hc sont inférieures à un seuil, le tableau 4 suivant peut être établi lors de la concomitance des événements compris dans une classe donnée :

Tableau 4. Tableau de concomitance houle/clapot.

\begin{tabular}{|c|c|c|c|c|c|}
\hline $\begin{array}{ll}H h & H c \\
\end{array}$ & $\begin{array}{c}C 2 \\
0,75-1,00\end{array}$ & $\begin{array}{c}C 3 \\
0,50-0,75\end{array}$ & $\begin{array}{c}C 4 \\
0,25-0,50\end{array}$ & $\begin{array}{c}C 5 \\
0-0,25\end{array}$ & $1 m<R$ \\
\hline$H 2 \quad 0,75-1,00$ & & & & & $0,75<R<1 m$ \\
\hline H3 $\quad 0,50-0,75$ & & & & & $0,50<R<0,75 m$ \\
\hline \begin{tabular}{|ll}
$H 4$ & $0,25-0,50$ \\
\end{tabular} & & & & & $0,25<R<0,50 m$ \\
\hline $0-0,25$ & & & & & $0<R<0,25 \mathrm{~m}$ \\
\hline
\end{tabular}

La probabilité de la concomitance de deux événements $H_{i}$ et $C_{i}$, pour $1<i<5$, peut être évaluée par excès une fois les probabilités des classes de houles et de clapots connues. Les événements n'étant pas indépendants, le majorant suivant peut être utilisé :

$\mathrm{P}\left(\mathrm{H}_{\mathrm{i}} \cap \mathrm{C}_{\mathrm{i}}\right) \leq \min \left(\mathrm{P}\left(\mathrm{H}_{\mathrm{i}}\right), \mathrm{P}\left(\mathrm{C}_{\mathrm{i}}\right)\right)$.

Par exemple, on peut obtenir une agitation résultante supérieure à un $1 \mathrm{~m}$ sans que la houle ou le clapot ne le soit (événement R1) pour les événements $\mathrm{C}_{\mathrm{i}} \cap \mathrm{H} 2, \mathrm{C} 2 \cap \mathrm{H}_{\mathrm{i}}$ et $\mathrm{C} 3 \cap \mathrm{H} 3$ avec $2<\mathrm{i}<5$ (voir tableau 4 ). On peut donc en déduire que :

$\mathrm{P}(\mathrm{R} 1)<\min \left(\mathrm{P}\left(\mathrm{C}_{\mathrm{i}}\right), \mathrm{P}(\mathrm{H} 2)\right)+\min \left(\mathrm{P}\left(\mathrm{H}_{\mathrm{i}}\right), \mathrm{P}(\mathrm{C} 2)\right)+\min (\mathrm{P}(\mathrm{C} 3), \mathrm{P}(\mathrm{H} 3))$

avec $(2<\mathrm{i}<5) ;$ avec $\min \left(\mathrm{P}\left(\mathrm{C}_{\mathrm{i}}\right), \mathrm{P}(\mathrm{H} 2)\right) \neq \min \left(\mathrm{P}\left(\mathrm{H}_{\mathrm{i}}\right), \mathrm{P}(\mathrm{C} 2)\right)$

De la même manière :

$\mathrm{P}(\mathrm{R} 1 \cup \mathrm{R} 2)<\min \left(\mathrm{P}\left(\mathrm{C}_{\mathrm{i}}\right), \mathrm{P}(\mathrm{H} 2)\right)+\min \left(\mathrm{P}\left(\mathrm{H}_{\mathrm{i}}\right), \mathrm{P}(\mathrm{C} 2)\right)+\min \left(\mathrm{P}\left(\mathrm{C}_{\mathrm{i}}\right), \mathrm{P}(\mathrm{H} 3)\right)+$ $\min \left(\mathrm{P}(\mathrm{C} 3), \mathrm{P}\left(\mathrm{H}_{\mathrm{i}}\right)\right)$ 
avec $(2<\mathrm{i}<5)$ pour $\min \left(\mathrm{P}\left(\mathrm{C}_{\mathrm{i}}\right), \mathrm{P}(\mathrm{H} 2)\right)$ et $\min \left(\mathrm{P}\left(\mathrm{H}_{\mathrm{i}}\right), \mathrm{P}(\mathrm{C} 2)\right)$; avec $(3<\mathrm{i}<5)$ pour $\min \left(\mathrm{P}\left(\mathrm{C}_{\mathrm{i}}\right), \mathrm{P}(\mathrm{H} 3)\right)$ et $\min \left(\mathrm{P}(\mathrm{C} 3), \mathrm{P}\left(\mathrm{H}_{\mathrm{i}}\right)\right)$; avec $\min \left(\mathrm{P}\left(\mathrm{C}_{\mathrm{i}}\right), \mathrm{P}(\mathrm{H} 3)\right) \neq \min \left(\mathrm{P}(\mathrm{C} 3), \mathrm{P}\left(\mathrm{H}_{\mathrm{i}}\right)\right) \neq$ $\min \left(\mathrm{P}\left(\mathrm{C}_{\mathrm{i}}\right), \mathrm{P}(\mathrm{H} 2)\right) \neq \min \left(\mathrm{P}\left(\mathrm{H}_{\mathrm{i}}\right), \mathrm{P}(\mathrm{C} 2)\right)$

$\mathrm{P}(\mathrm{R} 1 \cup \mathrm{R} 2 \cup \mathrm{R} 3)<\min \left(\mathrm{P}\left(\mathrm{C}_{\mathrm{i}}\right), \mathrm{P}(\mathrm{H} 2)\right)+\min \left(\mathrm{P}\left(\mathrm{H}_{\mathrm{i}}\right), \mathrm{P}(\mathrm{C} 2)\right)+\min \left(\mathrm{P}\left(\mathrm{C}_{\mathrm{i}}\right), \mathrm{P}(\mathrm{H} 3)\right)+$ $\min \left(\mathrm{P}(\mathrm{C} 3), \mathrm{P}\left(\mathrm{H}_{\mathrm{i}}\right)\right)+\min \left(\mathrm{P}\left(\mathrm{C}_{\mathrm{i}}\right), \mathrm{P}(\mathrm{H} 4)\right)+\min \left(\mathrm{P}(\mathrm{C} 4), \mathrm{P}\left(\mathrm{H}_{\mathrm{i}}\right)\right)$ avec $(2<\mathrm{i}<5)$ pour $\min \left(\mathrm{P}\left(\mathrm{C}_{\mathrm{i}}\right), \mathrm{P}(\mathrm{H} 2)\right)$ et $\min \left(\mathrm{P}\left(\mathrm{H}_{\mathrm{i}}\right), \mathrm{P}(\mathrm{C} 2)\right)$; avec $(3<\mathrm{i}<5)$ pour $\min \left(\mathrm{P}\left(\mathrm{C}_{\mathrm{i}}\right), \mathrm{P}(\mathrm{H} 3)\right)$ et $\min \left(\mathrm{P}(\mathrm{C} 3), \mathrm{P}\left(\mathrm{H}_{\mathrm{i}}\right)\right)$; avec $(4<\mathrm{i}<5)$ pour $\min \left(\mathrm{P}\left(\mathrm{C}_{\mathrm{i}}\right), \mathrm{P}(\mathrm{H} 4)\right)$ et $\min \left(\mathrm{P}(\mathrm{C} 4), \mathrm{P}\left(\mathrm{H}_{\mathrm{i}}\right)\right)$; avec $\min \left(\mathrm{P}\left(\mathrm{C}_{\mathrm{i}}\right), \mathrm{P}(\mathrm{H} 3)\right) \neq \min \left(\mathrm{P}(\mathrm{C} 3), \mathrm{P}\left(\mathrm{H}_{\mathrm{i}}\right)\right) \neq \min \left(\mathrm{P}\left(\mathrm{C}_{\mathrm{i}}\right), \mathrm{P}(\mathrm{H} 2)\right) \neq$ $\min \left(\mathrm{P}\left(\mathrm{H}_{\mathrm{i}}\right), \mathrm{P}(\mathrm{C} 2)\right) \neq \min \left(\mathrm{P}\left(\mathrm{C}_{\mathrm{i}}\right), \mathrm{P}(\mathrm{H} 4)\right) \neq \min \left(\mathrm{P}(\mathrm{C} 4), \mathrm{P}\left(\mathrm{H}_{\mathrm{i}}\right)\right)$

On pose d'après (2) : $\mathrm{P} 1=\min \left(\mathrm{P}\left(\mathrm{C}_{\mathrm{i}}\right), \mathrm{P}(\mathrm{H} 2)\right)+\min \left(\mathrm{P}\left(\mathrm{H}_{\mathrm{i}}\right), \mathrm{P}(\mathrm{C} 2)\right)+\min (\mathrm{P}(\mathrm{C} 3), \mathrm{P}(\mathrm{H} 3))$

D'où $\mathrm{P} 1=\mathrm{P}(\mathrm{H} 2)+\mathrm{P}(\mathrm{C} 2)+\min (\mathrm{P}(\mathrm{C} 3), \mathrm{P}(\mathrm{H} 3))$

On pose d'après (3): $\mathrm{P} 1+\mathrm{P} 2=\min \left(\mathrm{P}\left(\mathrm{C}_{\mathrm{i}}\right), \mathrm{P}(\mathrm{H} 2)\right)+\min \left(\mathrm{P}\left(\mathrm{H}_{\mathrm{i}}\right), \mathrm{P}(\mathrm{C} 2)\right)+$ $\min \left(\mathrm{P}\left(\mathrm{C}_{\mathrm{i}}\right), \mathrm{P}(\mathrm{H} 3)\right)+\min \left(\mathrm{P}(\mathrm{C} 3), \mathrm{P}\left(\mathrm{H}_{\mathrm{i}}\right)\right)$

D'où $\mathrm{P} 2=\max (\mathrm{P}(\mathrm{C} 3), \mathrm{P}(\mathrm{H} 3))$

On pose d'après (4): $\mathrm{P} 1+\mathrm{P} 2+\mathrm{P} 3=\min \left(\mathrm{P}\left(\mathrm{C}_{\mathrm{i}}\right), \mathrm{P}(\mathrm{H} 2)\right)+\min \left(\mathrm{P}\left(\mathrm{H}_{\mathrm{i}}\right), \mathrm{P}(\mathrm{C} 2)\right)+$ $\min \left(\mathrm{P}\left(\mathrm{C}_{\mathrm{i}}\right), \mathrm{P}(\mathrm{H} 3)\right)+\min \left(\mathrm{P}(\mathrm{C} 3), \mathrm{P}\left(\mathrm{H}_{\mathrm{i}}\right)\right)+\min \left(\mathrm{P}\left(\mathrm{C}_{\mathrm{i}}\right), \mathrm{P}(\mathrm{H} 4)\right)+\min \left(\mathrm{P}(\mathrm{C} 4), \mathrm{P}\left(\mathrm{H}_{\mathrm{i}}\right)\right)$

D'où $\mathrm{P} 3=\mathrm{P}(\mathrm{C} 4)+\mathrm{P}(\mathrm{H} 4)$

Ainsi à partir des probabilités des événements $\mathrm{H}_{\mathrm{i}}$ et $\mathrm{C}_{\mathrm{i}}$ (impact de la houle ou du clapot compris dans une classe donnée) (voir tableaux 2 et 3 pour le poste à quai $\mathrm{n}^{\circ} 6$ ) et du tableau 4, les probabilités $\mathrm{P} 1, \mathrm{P} 2$ et $\mathrm{P} 3$ et les probabilités des événements $\mathrm{Ht}>\mathrm{S}$ (pour $\mathrm{S}_{1}=1 \mathrm{~m}, \mathrm{~S}_{2}=0.75 \mathrm{~m}$ et $\mathrm{S}_{3}=0.5 \mathrm{~m}$ ), peuvent être calculées.

Pour le poste à quai 6 , d'après (5), (6) et (7), on obtient ainsi :

$\mathrm{P} 1=\mathrm{P}(\mathrm{C} 2)+\mathrm{P}(\mathrm{H} 2)+\min (\mathrm{P}(\mathrm{C} 3), \mathrm{P}(\mathrm{H} 3))=0,189+0,160+1,412=1,761 \%$

$\mathrm{P} 2=\max (\mathrm{P}(\mathrm{C} 3), \mathrm{P}(\mathrm{H} 3))=1,528 \%$

$\mathrm{P} 3=\mathrm{P}(\mathrm{C} 4)+\mathrm{P}(\mathrm{H} 4)=9,970+9,925=19,895 \%$

D'après (1), on obtient :

$\mathrm{P}(\mathrm{Ht}>1 \mathrm{~m})<\mathrm{P}(\mathrm{H} 1)+\mathrm{P}(\mathrm{C} 1)+\mathrm{P} 1=0,085+0,034+1,761=1,9 \%$

$\mathrm{P}(\mathrm{Ht}>0,75 \mathrm{~m})<\mathrm{P}(\mathrm{H} 1)+\mathrm{P}(\mathrm{C} 1)+\mathrm{P} 1+\mathrm{P} 2=0,085+0,034+1,761+1,528=3,4 \%$

$\mathrm{P}(\mathrm{H} \mathrm{t}>0,5 \mathrm{~m})<\mathrm{P}(\mathrm{H} 1)+\mathrm{P}(\mathrm{C} 1)+\mathrm{P} 1+\mathrm{P} 2+\mathrm{P} 3=0,085+0,034+1,761+1,528+19,895$

$=23,3 \%$

Ces probabilités ont été calculées de la même manière sur les autres postes à quai.

\section{Conclusion}

L'agitation a été évaluée sur les 12 postes à quai du bassin de Port 2000 pour les trois seuils de $1 \mathrm{~m}, 0,75 \mathrm{~m}$ et $0,5 \mathrm{~m}$. La méthodologie mise en œuvre n'a cependant permis qu'une évaluation par excès de l'agitation. De plus, il est à noter que la précision des 
résultats diminue avec le seuil. L'étude a montré que globalement l'impact des houles est prépondérant sur les postes 1 et de 6 à 12 (voir figure 2) alors que le clapot est prépondérant pour les postes 2 à 5 . La contribution de chaque phénomène seul est faible dans l'agitation totale; c'est en effet la combinaison de l'impact des houles et des clapots qui est à l'origine de la plus grande contribution dans l'agitation totale.

L'indisponibilité des postes à quai aux navettes fluviales reste cependant assez faible, en moyenne l'agitation sera supérieure à $1 \mathrm{~m}$ de 3 à 9 jours suivant les postes à quais. Les postes à quai les plus touchés sont les deux postes extérieurs ( ${ }^{\circ} 11$ et 12$)$, les houles du large se propageant en effet principalement dans l'axe du chenal d'accès.

\section{Références bibliographiques}

BISHOP C.T., DONELAN M.A. (1987). Measuring Waves with Pressure Transducers. Journal of Coastal Engineering, August 1986, Vol. 11, pp 309-328,.

BISHOP C.T., DONELAN M.A., KAHMA K.K. (1992). Shore Protection Manual's Wave Prediction Reviewed, Coastal Engineering, Vol. 17, pp 25-48. doi:10.1016/0378-3839(92)90012-J

DONELAN M.A. (1980). Similarity theory applied to the forecasting of wave heights, periods and directions. Proc. Of the 1980 Canadian Coastal Conf., Natl. Res. Council Canada, pp 47-61.

DONELAN M.A., SKAFEL M., GRABER H., LIU P., SCHWAB D., VENKATESH S. (1992). On the growth rate of wind-generated waves. Atmos.-Ocean, 30 (3), pp 457-478.

LEBRETON P., LE GUYADER C., LE LAMER P., MICHARD B. (2006). Évaluations des conditions de navigation pour les bateaux de navigation intérieure dans le bassin de Port 2000 au voisinage de l'écluse projetée. Grand Port du Havre, Rapport CETMEF/ DELCE, 15 p.

LE GUYADER C. (2007). Conditions de navigation des unités fluviales aux postes à quais et sur le trajet depuis l'écluse fluvio-maritime. Grand Port du Havre, Rapport CETMEF/ DELCE, $15 \mathrm{p}$.

PERHERIN C. (2008). Évaluation des conditions d'agitation dans le bassin Port 2000. Grand Port du Havre, Rapport CETMEF/ DELCE, 25 p. 